Karl-Franzens Universität Graz

Technische Universität Graz

Medizinische Universität Graz

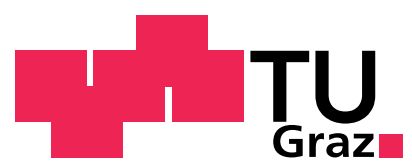

A parallel Newton-Krylov method for optimal control of the monodomain model in cardiac electrophysiology

Karl Kunisch Chamakuri Nagaiah Marcus Wagner 
SFB sponsors:

- Austrian Science Fund (FWF)

- University of Graz

- Graz University of Technology

- Medical University of Graz

- Government of Styria Das Land Steiermark

- City of Graz 


\title{
A parallel Newton-Krylov method for optimal control of the monodomain model in cardiac electrophysiology
}

Karl Kunisch, Chamakuri Nagaiah and Marcus Wagner ${ }^{\dagger}$

\begin{abstract}
This work addresses an optimal control approach for a model problem in cardiac electrophysiology. A rather complete treatment including the analysis of the model equations, derivation of the optimality system, description of its discretization and a numerical feasibility study in a parallel environment is provided.
\end{abstract}

Key words. PDE constrained optimization, monodomain equations, Rogers-McCulloch model, necessary optimality conditions, numerical solution, parallelization, receding horizon strategy.

AMS Subject Classification (2010). 35 G 31, 35 Q 92, 49 K 20, 49 M 05, 49 M 15, 65 Y 05, 92 C 30.

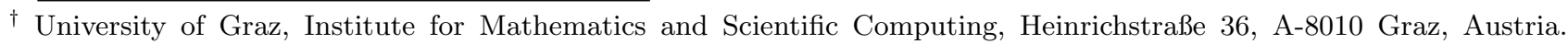
e-mails: karl.kunisch@uni-graz.at (Corresponding Author); nagaiah.chamakuri@uni-graz.at; marcus.wagner Q uni-graz.at 


\title{
A parallel Newton-Krylov method for optimal control of the monodomain model in cardiac electrophysiology
}

\author{
Karl Kunisch, Chamakuri Nagaiah and Marcus Wagner
}

\section{Introduction.}

The present paper is concerned with a parallel numerical method for the solution of an optimal control problem involving the monodomain equations of cardiac electrophysiology. Here the electrical behavior of the cardiac tissue is described by a single parabolic reaction-diffusion equation, which is coupled with an ODE. In their strong form, these equations may be stated as follows: ${ }^{01)}$

$$
\begin{aligned}
& \frac{\partial v}{\partial t}+I_{i o n}(v, w)-\operatorname{div}\left(\bar{\sigma}_{i} \nabla v\right)=I_{e} \quad \text { for a. a. }(x, t) \in \Omega \times[0, T] \\
& \frac{\partial w}{\partial t}+G(v, w)=0 \quad \text { for a. a. }(x, t) \in \Omega \times[0, T] \\
& \mathfrak{n}^{\mathrm{T}} \bar{\sigma}_{i} \nabla v=0 \text { for all }(x, t) \in \partial \Omega \times[0, T] ; \\
& v(x, 0)=v_{0}(x) \text { and } w(x, 0)=w_{0}(x) \quad \text { for a. a. } x \in \Omega .
\end{aligned}
$$

In this model, the heart muscle is assumed to occupy the spatial domain $\Omega \subset \mathbb{R}^{d}$. The anisotropic electrical properties of the heart tissue are described by a single conductivity tensor $\bar{\sigma}_{i}$, and the variables $v=v(x, t)$, $w=w(x, t)$ and $I_{e}=I_{e}(x, t)$ represent the transmembrane electrical potential, a so-called gating variable, which is related to the ion transport through the cell membranes, and the extracellular stimulation current, respectively. Since the heart tissue stays electrically isolated in the absence of a conductive bath, we impose weighted Neumann boundary conditions. Together with appropriate specifications of the ionic current $I_{i o n}$ and the function $G$ within (1.2), this system forms a well-accepted simplification for the full bidomain model of the electrical heart activity. ${ }^{02)}$ While conserving a number of essential features of the bidomain model, e. g. excitability phenomena, the monodomain equations can be solved at a much lower computational expense. ${ }^{03)}$

A strong motivation for the study of optimal control problems in relation with the equations of cardiac electrophysiology comes from the phenomenon of cardiac arrhythmia, a life-threatening situation, which is still recognized as a significant challenge for the clinical practice as well as for physiological research. The termination of a cardiac arrhythmia is best achieved by application of a strong electrical shock, a process called defibrillation. ${ }^{04)}$ From the viewpoint of applied mathematics, it is important to go beyond direct numerical simulation of typical defibrillation situations ${ }^{05)}$ by determining an applied electrical field as a control, to which the system responds according to specifications which are specified in an appropriately chosen cost functional. In this regard, a possible goal of optimization could be the dampening of sharp gradients of the transmembrane potential $v$ or even the dampening of the transmembrane potential at all.

01) For an introduction into the monodomain equations and the underlying bidomain model, see [SUNDNES/LinES/CAI/ NieLSEn/Mardal/TVeito 06], pp. $30 \mathrm{ff}$., and the references therein.

02) Cf. [ Nielsen/Ruud/Lines/Tveito 07] and [Potse/Dubé/Richer/Vinet/Gulrajani 06].

03) Due to the high spatio-temporal resolution required for resolving the wavefront propagation in the heart, the numerical solution of the full bidomain model is highly expensive, cf. e. g. [Vigmond/Weber dos Santos/Prassl/Deo/Plank 08 ] and [Weber dos Santos/Plank/Bauer/Vigmond 04]. Most time is consumed by the solution of the elliptic part of the model, cf. [Plank/Liebmann/Weber dos Santos/Vigmond/HaAse 07], which is absent in the monodomain approximation.

04) [KEENER/SNEYD 09] , pp. 583 ff. and $604 \mathrm{ff}$.

05) [ Anderson/Trayanova/Skouibine 00]. 
Based on these considerations, the following optimal control problem has been selected for numerical solution:

(P) $F\left(v, w, I_{e}\right)=\frac{1}{2} \int_{0}^{T} \int_{\Omega_{o b s}} v(x, t)^{2} d x d t+\frac{\alpha}{2} \int_{0}^{T} \int_{\Omega_{c o n}} I_{e}(x, t)^{2} d x d t \longrightarrow$ inf!

subject to the state equations $(1.1)-(1.4)$ in their weak formulation (see (2.1) - (2.3) below) and

the control restrictions $\left|I_{e}(x, t)\right| \leqslant R$ for a. a. $(x, t) \in \Omega_{\text {con }} \times[0, T]$ as well as

$I_{e}(x, t) \equiv 0$ for a. a. $(x, t) \in\left(\Omega \backslash \Omega_{c o n}\right) \times[0, T]$,

with $\alpha>0$ and $R>0$. In problem $(\mathrm{P}), v$ and $w$ are the state variables while $I_{e}$ acts as a locally supported distributed control. The objective functional consists of a data term, which enforces the dampening of excitation waves of the transmembrane potential $v$, and a quadratic regularization term for the control variable. The behavior of $v$ will be observed within a subdomain $\Omega_{o b s} \subset \Omega$, and the control will be applied at a subdomain $\Omega_{\text {con }} \subset \Omega$ of comparatively small measure. The control restriction (1.6) reflects the obvious fact that one cannot apply arbitrarily large electrical stimulations to living tissue without damaging it. For $I_{i o n}$ and $G$, we specify the Rogers-McCulloch model (see Subsection 2.b) below).

Two case studies, corresponding to different initial conditions for $v$, will be performed. In our first experiment, we aim for dampening of a single excitation wave. In this particular case, the authors already obtained numerical results using a different, non-parallel implementation. ${ }^{06}$ ) Consequently, the problem can serve as a test case for measuring the efficiency of the parallelized approach. In our second experiment, the initial condition corresponds to a situation of cardiac arrhythmia, which is characterized by the presence of a reentrant excitation wave, and the goal is to terminate this wave. In both cases, we work on a two-dimensional spatial domain. An important feature for the choice of the numerical test cases is the size of the subdomain $\Omega_{c o n}$, on which the control is exercised. Achieving the design objective, in our case the extinction of waves, is increasingly more difficult as the size of $\Omega_{c o n}$ is decreased.

As in other PDE constrained optimization problems, the discretization of the first-order necessary optimality conditions results in a nonlinear system of considerable size and complexity. In the literature, systems of this type have been accessed with higher-order optimization techniques, ${ }^{07)}$ adaptive techniques ${ }^{08)}$ as well as with parallelization strategies. ${ }^{09)}$ In the present investigation, pursuing the strategy "optimize before discretize", 10) we combine a Krylov method for the solution of the primal and adjoint equations with an inexact Newton method for the minimization of the reduced cost functional ${ }^{11)}$ and focus on the parallelization of the algorithm, which reduces the computation time to $4.2 \%$ compared to the sequential code. In our second case study, this approach has been combined with a receding horizon strategy, which allows for long-time simulations. As a result, the re-entrant wave has been completely removed after a simulation time of 180 ms, which took approximately 1.25 hours of CPU time on a cluster with 32 processors.

Concerning the optimal control of the monodomain or even the bidomain equations, fairly little work is available in the literature as yet. The analysis of $(\mathrm{P})$ with the Rogers-McCulloch model was given in [ KUnISCH/WAGNER 11 ], comprising a rigorous proof of the well-posedness of the control-to-state mapping as well as of the firstorder optimality conditions. ${ }^{12)}$ The studies [NAGAiah/Kunisch/Plank 09] and [NAGAIAH/Kunisch 11] are concerned with the numerical solution of control problems closely related with $(\mathrm{P})$. In these papers, the calculations have been performed on a two-dimensional spatial domain as well. To the best of our knowledge, the

06) Cf. [Nagaiah/Kunisch/Plank 09] and [Nagaiah/Kunisch 11].

07) [HinZE/Kunisch 01].

08) We mention e. g. [Hintermüller/Hoppe 08], [Meidner/Vexler 07] and [Nagaiah/Kunisch 11].

09) [Biros/Ghattas 05].

10) A detailed comparison between the "discretize before optimize" and the "optimize before discretize" approaches to the solution of $(\mathrm{P})$ in the case $\Omega \subset \mathbb{R}^{1}$ has been provided in [NAGAIAH/KUnisCH/Plank 10] .

11) See e. g. [Biegler/Ghattas/Heinkenschloss/Keyes/van Bloemen WaAnders 07] .

12) Under analytical viewpoints, a related control problem involving the FitzHugh-Nagumo model and Dirichlet boundary conditions has been studied in [Brandẽo/FERnÁndez-Cara/Magalhães/Rojas-Medar 08] . 
present work is the first attempt at a parallelized numerical solution of control problems for the monodomain system.

The paper is organized as follows. In Section 2, we state the weak formulation of the monodomain system together with the Rogers-McCulloch model and provide an existence and uniqueness theorem. In Section 3, we start with the precise statement of the control problem $(\mathrm{P})$, subsequently turning to the formulation of the necessary optimality conditions and the description of the first variation of the reduced cost functional. In Section 4, we describe the numerical approach, comprising the semi-discretization of the primal and adjoint equations in space, the solution of the resulting ODE system by decoupling, the iteration scheme for the update of the control variable as well as the parallel implementation. Short remarks about the receding horizon strategy will be included. Section 5 is devoted to the description of the numerical experiments as well as to the presentation and discussion of the results. Finally, the parallel algorithm will be listed in an appendix.

\section{Notations.}

We denote by $L^{p}(\Omega)$ the space of functions, which are in the $p$ th power integrable $(1 \leqslant p<\infty)$, or are measurable and essentially bounded $(p=\infty)$, and by $W^{1, p}(\Omega)$ the Sobolev space of functions $\psi: \Omega \rightarrow \mathbb{R}$ which, together with their first-order weak partial derivatives, belong to the space $L^{p}(\Omega)(1 \leqslant p<\infty)$. For spaces of Bochner integrable mappings, e. g. $L^{2}\left[(0, T), W^{1,2}(\Omega)\right]$, we refer to [KUNISCH/WAGNER 11] p. $29 \mathrm{f} . \Omega_{T}$ is an abbreviation for $\Omega \times[0, T]$. The gradient $\nabla$ is always taken only with respect to the spatial variables $x \cdot \mathbb{1}_{\mathrm{A}}$ denotes the characteristic function of the set $\mathrm{A} \subseteq \Omega$. The abbreviation " $(\forall) t \in \mathrm{A}$ " has to be read as "for almost all $t \in \mathrm{A}$ " or "for all $t \in \mathrm{A}$ except a Lebesgue null set", and the symbol $\mathfrak{o}$ denotes, depending on the context, the zero element or the zero function of the underlying space.

\section{The two-dimensional monodomain model.}

\section{a) Weak formulation of the monodomain equations.}

The rigorous analysis of the optimal control problem $(\mathrm{P})$ is based on a weak solution concept for the monodomain equations $(1.1)-(1.4)$. The weak formulation of the system reads as follows:

$$
\begin{aligned}
& \int_{\Omega}\left(\frac{\partial v}{\partial t}+I_{i o n}(v, w)\right) \psi d x+\int_{\Omega} \nabla \psi^{\mathrm{T}} \bar{\sigma}_{i} \nabla v d x=\int_{\Omega} I_{e} \psi d x \quad \forall \psi \in W^{1,2}(\Omega)(\forall) t \in[0, T] ; \\
& \int_{\Omega}\left(\frac{\partial w}{\partial t}+G(v, w)\right) \psi d x=0 \quad \forall \psi \in L^{2}(\Omega)(\forall) t \in[0, T] ; \\
& v(x, 0)=v_{0}(x) \text { and } w(x, 0)=w_{0}(x) \quad(\forall) x \in \Omega .
\end{aligned}
$$

Definition 2.1. (Weak solution of the monodomain system) ${ }^{13)}$ Let $T>0$. A pair $(v, w)$ is called a weak solution of the monodomain system $(2.1)-(2.3)$ on $[0, T]$ iff $v$ and $w$ satisfy the system on $[0, T]$ in the distributional sense and obey the initial conditions. Moreover, the functions belong to the spaces

$$
\begin{aligned}
& v \in C^{0}\left[[0, T], L^{2}(\Omega)\right] \cap L^{2}\left[(0, T), W^{1,2}(\Omega)\right] \cap L^{p}\left(\Omega_{T}\right) \text { with } 2 \leqslant p \leqslant 6 ; \\
& w \in C^{0}\left[[0, T], L^{2}(\Omega)\right] .
\end{aligned}
$$

\section{b) The Rogers-McCulloch model for the ionic current.}

In the literature, a variety of ionic models describing the cardiac electrical phenomena have been proposed. For the present study, we specify a simplified two-variable model, namely the Rogers-McCulloch model. ${ }^{14)}$ In this model, the ionic current term is the sum of a cubic polynomial in $v$ and the multiplicative coupling term

13) See [Bourgault/Coudière/Pierre 09], p. 472, Definition 26.

14) Cf. [Rogers/McCulloch 94]. It can be understood as a slight generalization of the most frequently considered FitzHugh-Nagumo model. 
$\eta_{1} v w$ while the effects of the ion transport through the cell membranes will be described in a cumulative way through a single gating variable $w$. More precisely, $I_{i o n}$ and $G$ are expressed as

$$
\begin{aligned}
& I_{\text {ion }}(v, w)=\eta_{0} \cdot v\left(1-\frac{v}{v_{t h}}\right)\left(1-\frac{v}{v_{p k}}\right)+\eta_{1} v w \\
& G(v, w)=\eta_{2}\left(\eta_{3} w-\frac{v}{v_{p k}}\right),
\end{aligned}
$$

where $\eta_{0}, \eta_{1}, \eta_{2}, \eta_{3}>0$ are positive coefficients, $v_{t h}>0$ is a threshold potential, and $v_{p k}>0$ is a peak potential. Consequently, the gating variable obeys the linear ODE

$$
\frac{\partial w}{\partial t}+\eta_{2} \eta_{3} w=\frac{\eta_{2}}{v_{p k}} v
$$

\section{c) Existence and uniqueness of weak solutions.}

From [KunisCH/WAGNER 11], we take the following existence and uniqueness theorem for the weak monodomain system $(2.1)-(2.3)$ :

Theorem 2.2. (Existence and uniqueness of weak solutions in relation to feasible controls) ${ }^{15)}$ Assume that $\Omega \subset \mathbb{R}^{2}$ is a bounded strong Lipschitz domain, and $\bar{\sigma}_{i}: \operatorname{cl}(\Omega) \rightarrow \mathbb{R}^{2 \times 2}$ is a symmetric, positive definite matrix function with $L^{\infty}(\Omega)$-entries, which obeys a uniform ellipticity condition. Then for any choice of initial values $v_{0} \in L^{2}(\Omega), w_{0} \in L^{4}(\Omega)$ and right-hand side $I_{e} \in L^{\infty}\left[(0, T), L^{2}(\Omega)\right]$, the monodomain system (2.1) - (2.3) admits a unique weak solution $(v, w)$ in the sense of Definition 2.1. on [0,T]. The solution satisfies the a priori estimate

$$
\begin{gathered}
\|v\|_{C^{0}\left[[0, T), L^{2}(\Omega)\right]}^{2}+\|v\|_{L^{2}\left[(0, T), W^{1,2}(\Omega)\right]}^{2}+\|v\|_{L^{4}\left(\Omega_{T}\right)}^{4}+\|\partial v / \partial t\|_{L^{4 / 3}\left[(0, T),\left(W^{1,2}(\Omega)\right)^{*}\right]}^{4 / 3} \\
\quad+\|w\|_{C^{0}\left[[0, T), L^{2}(\Omega)\right]}^{2}+\|\partial w / \partial t\|_{L^{2}\left[(0, T),\left(W^{1,2}(\Omega)\right)^{*}\right]}^{2} \\
\leqslant C \cdot\left(1+\left\|\Phi_{0}\right\|_{L^{2}(\Omega)}^{2}+\left\|W_{0}\right\|_{L^{4}(\Omega)}^{4}+\left\|I_{e}\right\|_{L^{2}\left[(0, T), L^{2}(\Omega)\right]}^{2}\right) .
\end{gathered}
$$

Proof. Note first that [Kunisch/Wagner 11], p. 5 f., Theorems 2.5. - 2.8., remain valid even in the twodimensional case $\Omega \subset \mathbb{R}^{2}$. Then we must only check whether the functions $I_{i o n}$ and $G$ satisfy the growth conditions from [Kunisch/Wagner 11], p. 4, Assumption 2.3., 4). Obviously, $I_{i o n}(v, w)=F_{1}(v)+F_{2}(v) w$, and $G(v, w)=G_{1}(v)+g_{2} w$ with the continuous functions

$$
F_{1}(v)=\frac{\eta_{0}}{v_{t h} v_{p k}} v^{3}-\frac{\eta_{0}\left(v_{p k}+v_{t h}\right)}{v_{t h} v_{p k}} v^{2}+\eta_{0} v ; \quad F_{2}(v)=\eta_{1} v ; \quad G_{1}(v)=-\frac{\eta_{2}}{v_{p k}} v
$$

and $g_{2}=\eta_{2} \eta_{3}$. Using Young's inequality, we find with $p=4$ :

$$
\begin{aligned}
& \left|F_{1}(v)\right| \leqslant\left(\frac{2 \eta_{0}}{3}+\frac{\eta_{0}\left(v_{p k}+v_{t h}\right)}{3 v_{t h} v_{p k}}\right)+\left(\frac{\eta_{0}}{v_{t h} v_{p k}}+\frac{2 \eta_{0}\left(v_{p k}+v_{t h}\right)}{3 v_{t h} v_{p k}}+\frac{\eta_{0}}{3}\right)|v|^{p-1} ; \\
& \left|F_{2}(v)\right|=\eta_{1}|v|^{(p-2) / 2} ; \quad\left|G_{1}(v)\right| \leqslant \frac{\eta_{2}}{2 v_{p k}}+\frac{\eta_{2}}{2 v_{p k}}|v|^{p / 2} .
\end{aligned}
$$

In order to confirm the remaining condition, we abbreviate

$$
\begin{array}{r}
H(v, w)=\varrho I_{\text {ion }}(v, w) v+G(v, w) w=\frac{\varrho \eta_{0}}{v_{t h} v_{p k}} v^{4}-\frac{\varrho \eta_{0}\left(v_{p k}+v_{t h}\right)}{v_{t h} v_{p k}} v^{3}+\varrho \eta_{0} v^{2}+\varrho \eta_{1} v^{2} w \\
-\frac{\eta_{2}}{v_{p k}} v w+\eta_{2} \eta_{3} w^{2} .
\end{array}
$$

Considering the estimates

$$
\begin{aligned}
& |v w| \geqslant-\frac{1}{2 \varrho} \varrho|v|^{2}-\frac{1}{2}|w|^{2} ; \quad\left|v^{2} w\right| \geqslant-\frac{\varepsilon_{1}}{2}|v|^{4}-\frac{1}{2 \varepsilon_{1}}|w|^{2} ; \\
& \left|\frac{\eta_{0}\left(v_{p k}+v_{t h}\right)}{v_{t h} v_{p k}} v^{3}\right| \geqslant-\frac{1}{4\left(\varepsilon_{2}\right)^{4}}\left(\frac{\eta_{0}\left(v_{p k}+v_{t h}\right)}{v_{t h} v_{p k}}\right)^{4}-\frac{3\left(\varepsilon_{2}\right)^{4 / 3}}{4}|v|^{4},
\end{aligned}
$$

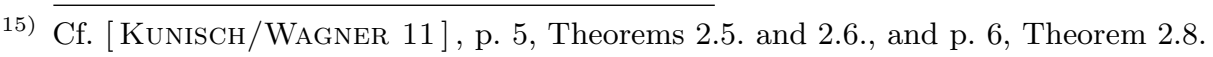


which have been obtained using the generalized Cauchy's and Young's inequalities with arbitrary $\varepsilon_{1}, \varepsilon_{2}>0,{ }^{16)}$ we arrive at

$$
\begin{array}{r}
H(v, w) \geqslant\left(\frac{\varrho \eta_{0}}{v_{t h} v_{p k}}-\frac{\varepsilon_{1}}{2}-\frac{3\left(\varepsilon_{2}\right)^{4 / 3}}{4}\right)|v|^{p}+\left(\eta_{0}+\eta_{2} \eta_{3}-\frac{\eta_{2}}{2 \varrho v_{p k}}-\frac{\eta_{2}}{2 v_{p k}}-\frac{1}{2 \varepsilon_{1}}\right)\left(\varrho|v|^{2}+|w|^{2}\right) \quad(2.18) \\
-\frac{1}{4\left(\varepsilon_{2}\right)^{4}}\left(\frac{\eta_{0}\left(v_{p k}+v_{t h}\right)}{v_{t h} v_{p k}}\right)^{4},
\end{array}
$$

and by appropriate choice first of $\varepsilon_{1}$ and $\varepsilon_{2}$ and subsequently of $\varrho$, it may be accomplished that on the righthand side of (2.17), the first coefficient becomes positive and the second one becomes negative. The proof is complete.

Remark. The compatibility condition $\int_{\Omega} I_{e}(x, t) d x=0(\forall) t \in[0, T]$, which would be a mandatory assumption for the solvability of the full bidomain system, ${ }^{17)}$ plays no role for the existence of solutions of the monodomain equations.

\section{The optimal control problem.}

\section{a) Statement of the problem.}

More precisely, the control problem (1.5) - (1.7) may be stated as follows:

$$
\begin{aligned}
& F\left(v, w, I_{e}\right)=\frac{1}{2} \int_{0}^{T} \int_{\Omega_{o b s}} v(x, t)^{2} d x d t+\frac{\alpha}{2} \int_{0}^{T} \int_{\Omega_{c o n}} I_{e}(x, t)^{2} d x d t \longrightarrow \inf ! \\
& E_{1}\left(v, w, I_{e}\right)=\int_{\Omega}\left(\frac{\partial v(t)}{\partial t}+I_{i o n}(v(t), w(t))-I_{e}(t)\right) \psi d x \\
& +\int_{\Omega} \nabla \psi^{\mathrm{T}} \bar{\sigma}_{i} \nabla v(t) d x=0 \quad \forall \psi \in W^{1,2}(\Omega)(\forall) t \in[0, T] ; \\
& E_{2}(v, w)=\int_{\Omega}\left(\frac{\partial w(t)}{\partial t}+G(v(t), w(t))\right) \psi d x=0 \quad \forall \psi \in L^{2}(\Omega) \quad(\forall) t \in[0, T] ; \\
& E_{3}(v)=v(x, 0)-v_{0}(x)=0 \quad(\forall) x \in \Omega ; \\
& E_{4}(w)=w(x, 0)-w_{0}(x)=0 \quad(\forall) x \in \Omega ; \\
& I_{e} \in \mathcal{C}=\left\{z \in L^{\infty}\left[(0, T), L^{2}(\Omega)\right]|| z(x, t) \mid \leqslant R(\forall)(x, t) \in \Omega_{c o n} \times[0, T] ;\right. \\
& \left.z(x, t) \equiv 0(\forall)(x, t) \in\left(\Omega \backslash \Omega_{c o n}\right) \times[0, T]\right\} .
\end{aligned}
$$

We suppose that the data satisfy the assumptions of Theorem 2.2. The numbers $T>0, \alpha>0$ and $R>0$ are fixed. The functions $I_{i o n}(v, w)$ and $G(v, w)$ will be specified according to the Rogers-McCulloch model from Subsection 2.b). The domains of definition and the ranges of the operators $F, E_{1}, \ldots, E_{4}$ are chosen as follows:

$$
\begin{aligned}
& F: \mathrm{X}_{1} \times \mathrm{X}_{2} \times \mathrm{X}_{3} \rightarrow \mathbb{R} \quad \text { with } \\
& \mathrm{X}_{1}=L^{2}\left[(0, T), W^{1,2}(\Omega)\right] ; \quad \mathrm{X}_{2}=L^{2}\left[(0, T), L^{2}(\Omega)\right] ; \quad \mathrm{X}_{3}=L^{\infty}\left[(0, T), L^{2}(\Omega)\right] ; \\
& E_{1}: \mathrm{X}_{1} \times \mathrm{X}_{2} \times \mathrm{X}_{3} \rightarrow L^{4 / 3}\left[(0, T),\left(W^{1,2}(\Omega)\right)^{*}\right] ; \\
& E_{2}: \mathrm{X}_{1} \times \mathrm{X}_{2} \rightarrow L^{2}\left[(0, T),\left(L^{2}(\Omega)\right)^{*}\right] ; \\
& E_{3}: \mathrm{X}_{1} \rightarrow L^{2}(\Omega) ; \\
& E_{4}: \mathrm{X}_{2} \rightarrow L^{2}(\Omega) .
\end{aligned}
$$

Theorem 3.1. (Existence of global minimizers) Under the assumptions of Theorem 2.2., the problem (P) admits a global minimizer $\left(\hat{v}, \hat{w}, \hat{I}_{e}\right) \in \mathrm{X}_{1} \times \mathrm{X}_{2} \times \mathrm{X}_{3}$.

16) $[$ Evans 98], p. 622.

17) See [Veneroni 09], p. 852. 
Proof. This is an immediate consequence of [Kunisch/WAGner 11], p. 17 f., Theorem 3.3., since the set $\mathcal{C}$ of admissible controls according to (3.6) can be considered as a closed, convex, weak ${ }^{*}$-sequentially compact subset of $L^{\infty}\left[(0, T), L^{2}\left(\Omega_{c o n}\right)\right]$, which may be continuously imbedded into $L^{\infty}\left[(0, T), L^{2}(\Omega)\right]$ by zero extension.

\section{b) The first-order necessary optimality conditions.}

We search for weak local minimizers according to the following definition:

Definition 3.2. (Weak local minimizer) A triple $\left(\hat{v}, \hat{w}, \hat{I}_{e}\right)$, which is feasible in $(\mathrm{P})$, is called a weak local minimizer of $(\mathrm{P})$ iff there exists a number $\varepsilon>0$ such that for all admissible $\left(v, w, I_{e}\right)$ the conditions

$$
\|v-\hat{v}\|_{\mathrm{X}_{1}} \leqslant \varepsilon,\|w-\hat{w}\|_{\mathrm{X}_{2}} \leqslant \varepsilon,\left\|I_{e}-\hat{I}_{e}\right\|_{\mathrm{X}_{3}} \leqslant \varepsilon
$$

imply the relation $F\left(\hat{v}, \hat{w}, \hat{I}_{e}\right) \leqslant F\left(v, w, I_{e}\right)$.

The existence of at least one weak local minimizer for $(\mathrm{P})$ is confirmed by Theorem 3.1. After introduction of the Lagrange function

$\mathcal{L}\left(v, w, I_{e}, p_{1}, p_{2}, p_{3}, p_{4}\right)=F\left(v, w, I_{e}\right)+\left\langle p_{1}, E_{1}\left(v, w, I_{e}\right)\right\rangle+\left\langle p_{2}, E_{2}(v, w)\right\rangle+\left\langle p_{3}, E_{3}(v)\right\rangle+\left\langle p_{4}, E_{4}(w)\right\rangle$

with multipliers $p_{1} \in L^{4}\left[(0, T), W^{1,2}(\Omega)\right], p_{2} \in L^{2}\left[(0, T), L^{2}(\Omega)\right]$, and $p_{3}, p_{4} \in\left(L^{2}(\Omega)\right)^{*}$, we obtain the following system of first-order necessary optimality conditions for $(\mathrm{P})$ :

Theorem 3.3. (Necessary optimality conditions for $(\mathrm{P})$ ) Assume that $\Omega \subset \mathbb{R}^{2}$ is a bounded domain with $C^{2, \varepsilon}$-boundary, $0<\varepsilon \leqslant 1$, and $\bar{\sigma}_{i}: \operatorname{cl}(\Omega) \rightarrow \mathbb{R}^{2 \times 2}$ is a symmetric, positive definite matrix function with $W^{1, \infty}(\Omega)$-coefficients whose boundary values belong even to $C^{1, \varepsilon}(\partial \Omega)$. $\bar{\sigma}_{i}$ satisfies a uniform ellipticity condition. Further, let the initial values belong to the following spaces: $v_{0} \in W^{2,2}(\Omega), w_{0} \in L^{\infty}(\Omega)$. ${ }^{18)}$

If a triple $\left(\hat{v}, \hat{w}, \hat{I}_{e}\right) \in L^{2}\left[(0, T), W^{1,2}(\Omega)\right] \times L^{2}\left[(0, T), L^{2}(\Omega)\right] \times L^{\infty}\left[(0, T), L^{2}(\Omega)\right]$ is a weak local minimizer of $(\mathrm{P})$, then there exist multipliers $p_{1}=p \in L^{r}\left[(0, T), W^{2, r}(\Omega)\right] \cap W^{1, r}\left[(0, T), L^{r}(\Omega)\right]$ and $p_{2}=q \in C^{1}\left[(0, T), L^{r}(\Omega)\right] \cap C^{0}\left[[0, T], L^{r}(\Omega)\right], 4<r<6$, satisfying together with $\left(\hat{v}, \hat{w}, \hat{I}_{e}\right)$ the optimality condition ${ }^{19)}$

$$
\int_{0}^{T} \int_{\Omega_{c o n}}\left(\alpha \hat{I}_{e}(x, t)-p(x, t)\right)\left(I_{e}(x, t)-\hat{I}_{e}(x, t)\right) d x d t \geqslant 0 \quad \forall I_{e} \in \mathcal{C}
$$

as well as the adjoint system

$$
\begin{aligned}
& -\frac{\partial p}{\partial t}-\nabla \cdot\left(\bar{\sigma}_{i} \nabla p\right)+\frac{\partial I_{\text {ion }}}{\partial v}(\hat{v}, \hat{w}) p=-\frac{\partial G}{\partial v}(\hat{v}, \hat{w}) q-\mathbb{1}_{\Omega_{o b s}} \cdot \hat{v} ; \\
& -\frac{\partial q}{\partial t}+\frac{\partial G}{\partial w}(\hat{v}, \hat{w}) q=-\frac{\partial I_{i o n}}{\partial w}(\hat{v}, \hat{w}) p ; \\
& \mathfrak{n}^{\mathrm{T}} \bar{\sigma}_{i} \nabla p=0 \forall(x, t) \in \partial \Omega \times[0, T] ; \quad p(x, T)=0(\forall) x \in \Omega ; \quad q(x, T)=0(\forall) x \in \Omega,
\end{aligned}
$$

where

$$
\begin{aligned}
& \frac{\partial I_{i o n}}{\partial v}(\hat{v}, \hat{w})=\frac{3 \eta_{0}}{v_{t h} v_{p k}} \hat{v}^{2}-\frac{2 \eta_{0}\left(v_{p k}+v_{t h}\right)}{v_{t h} v_{p k}} \hat{v}+\eta_{0}+\eta_{1} \hat{w} ; \quad \frac{\partial G}{\partial v}(\hat{v}, \hat{w}) \equiv-\frac{\eta_{2}}{v_{p k}} \\
& \frac{\partial G}{\partial w}(\hat{v}, \hat{w}) \equiv \eta_{2} \eta_{3} ; \quad \frac{\partial I_{i o n}}{\partial w}(\hat{v}, \hat{w})=\eta_{1} \hat{v}
\end{aligned}
$$

The functions $p$ and $q$ solve the adjoint system in the weak as well as in the strong sense.

18) The assumptions, which ensure the solvability of the adjoint system, have been taken over from [KUNISCH/ WAGNER 11 ] , p. 19, Theorem 3.5. They may be surely further relaxed since the cited theorem is based on a maximal regularity result.

19) For the multipliers $p_{3}$ and $p_{4}$ from (3.13), the zero functions may be chosen. 
Proof. Denote the integrand within the objective by $r\left(v, w, I_{e}\right)=0.5 v^{2}+0.5 \alpha I_{e}^{2}$. We may apply [KunisCH/ WAGNer 11], p. 20, Theorem 3.7., since $\partial r(\hat{v}, \hat{w}) / \partial v=\mathbb{1}_{\Omega_{o b s}} \hat{v}$ can be imbedded into the space $L^{\infty}[[0, T]$, $\left.W^{1,2}(\Omega)\right] \hookrightarrow L^{r}\left(\Omega_{T}\right)$ for arbitrary $4<r<6$, and the same is true for $\partial r(\hat{v}, \hat{w}) / \partial w=\mathfrak{o}$. Consequently, the optimality condition and the adjoint system take the claimed form. Note that, in (3.14), the integration may be restricted to $\Omega_{c o n}$ since all feasible controls vanish outside of this subdomain.

Corollary 3.4. (Pointwise formulation of the optimality condition) ${ }^{20)}$ The optimality condition (3.14) from Theorem 3.3. implies the following condition, which holds a. e. pointwise:

$$
\left(\alpha \hat{I}_{e}(x, t)-p(x, t)\right) \hat{I}_{e}(x, t)=\operatorname{Min}_{-R \leqslant \gamma \leqslant R}\left(\alpha \hat{I}_{e}(x, t)-p(x, t)\right) \gamma \quad(\forall)(x, t) \in \Omega_{c o n} \times[0, T]
$$

and $\hat{I}_{e}(x, t)=0$ elsewhere. Consequently, we have for almost all $(x, t) \in \Omega_{c o n} \times[0, T]$ :

$$
\hat{I}_{e}(x, t)=\left\{\begin{array}{c|l}
-R & \mid p(x, t)<-\alpha R \\
p(x, t) / \alpha & \mid p(x, t) \in[-\alpha R, \alpha R] ; \\
R & \mid p(x, t)>\alpha R
\end{array}\right.
$$

To sum up, the system of the first-order necessary optimality conditions consists of the initial-boundary value problem $(1.1)-(1.4)$ for the state equations, the terminal-boundary value problem $(3.15)-(3.17)$ for the adjoint equations and the optimality condition (3.21).

\section{c) The reduced cost functional.}

In view of Theorem 2.2., problem $(\mathrm{P})$ admits a well-defined control-to-state mapping $\mathcal{C} \ni I_{e} \longmapsto\left(v\left(I_{e}\right)\right.$, $\left.w\left(I_{e}\right)\right)$. Consequently, the objective (3.1) within (P) may be rewritten as

$$
\widetilde{F}\left(I_{e}\right)=\frac{1}{2} \int_{0}^{T} \int_{\Omega_{o b s}} v\left(I_{e}(x, t)\right)^{2} d x d t+\frac{\alpha}{2} \int_{0}^{T} \int_{\Omega_{c o n}} I_{e}(x, t)^{2} d x d t .
$$

As a consequence of the first-order necessary optimality conditions, the first variation $\delta^{+} \widetilde{F}$ of the reduced cost functional at a weak local minimizer $\hat{I}_{e} \longmapsto\left(v\left(\hat{I}_{e}\right), w\left(\hat{I}_{e}\right)\right)$ may be represented as

$$
\left\langle\delta^{+} \widetilde{F}\left(\hat{I}_{e}\right), I_{e}-\hat{I}_{e}\right\rangle=\int_{0}^{T} \int_{\Omega_{c o n}}\left(\alpha \hat{I}_{e}(x, t)-p(x, t)\right)\left(I_{e}(x, t)-\hat{I}_{e}(x, t)\right) d x d t
$$

where $p$ is a multiplier related to $\left(v\left(\hat{I}_{e}\right), w\left(\hat{I}_{e}\right), \hat{I}_{e}\right)$ according to Theorem $3.3 .{ }^{21)}$

\section{The numerical approach.}

\section{a) Semi-discretization in space of the primal and adjoint equations.}

For the following calculations, we introduce a spatial grid and a sequence of uniform time steps. In order to solve the system of the first-order necessary optimality conditions, we will consider first a semi-discrete analogue of the full system, discretizing in space using a standard finite element method with piecewise-linear continuous elements. Recall the weak formulation of the monodomain equations, which is given by

$$
\begin{aligned}
& \left\langle\frac{\partial v}{\partial t}+I_{i o n}(v, w)-I_{e}, \psi\right\rangle=-\left\langle\bar{\sigma}_{i} \nabla v, \nabla \psi\right\rangle \quad \forall \psi \in W^{1,2}(\Omega)(\forall) t \in[0, T] ; \\
& \left\langle\frac{\partial w}{\partial t}, \psi\right\rangle=-\langle G(v, w), \psi\rangle \quad \forall \psi \in L^{2}(\Omega)(\forall) t \in[0, T] ; \\
& v(x, 0)=v_{0}(x) \quad(\forall) x \in \Omega ; \quad w(x, 0)=w_{0}(x) \quad(\forall) x \in \Omega .
\end{aligned}
$$

20) $\overline{\text { [KUnisch/Wagner 11] , p. 20, Corollary } 3.8 .}$

21) Cf. [Ito/Kunisch 08], p. xii f., and [Kunisch/Wagner 11], p. 28 f., Proof of Theorem 3.7., Step 4. 
Introducing a finite-dimensional subspace $\mathrm{V}_{h} \subset W^{1,2}(\Omega) \subset L^{2}(\Omega)$, which is generated by the set $\left\{\omega_{j}\right\}_{j=1}^{N}$ of piecewise linear basis functions adapted to the spatial grid, the approximate solutions of $(4.1)-(4.3)$ can be expressed as $v(x, t) \approx \sum_{j=1}^{N} v_{j}(t) \omega_{j}(x)$ and $w(x, t) \approx \sum_{j=1}^{N} w_{j}(t) \omega_{j}(x)$. The control variable will be discretized with respect to the same basis as $I_{e}(x, t) \approx \sum_{j=1}^{N} I_{e, j}(t) \omega_{j}(x)$. Consequently, from (4.1) - (4.3) we get the following system of Ritz equations:

$$
\begin{aligned}
& \boldsymbol{M} \frac{d \boldsymbol{v}}{d t}=-\boldsymbol{A} \boldsymbol{v}-\boldsymbol{I}_{i o n}(\boldsymbol{v}, \boldsymbol{w})+\boldsymbol{M} \boldsymbol{I}_{e} \\
& \frac{d \boldsymbol{w}}{d t}=-\eta_{2} \eta_{3} \boldsymbol{w}+\frac{\eta_{2}}{v_{p k}} \boldsymbol{v} ; \quad \boldsymbol{v}(0)=\boldsymbol{v}_{0} ; \quad \boldsymbol{w}(0)=\boldsymbol{w}_{0}
\end{aligned}
$$

where $\boldsymbol{v}=\boldsymbol{v}(t), \boldsymbol{w}=\boldsymbol{w}(t)$ and $\boldsymbol{I}_{e}=\boldsymbol{I}_{e}(t)$ are the vectors of Ritz coefficients, $\boldsymbol{M}=\left\{\left\langle\omega_{j}, \omega_{k}\right\rangle\right\}_{j, k=1}^{N}$ is the mass matrix, $\boldsymbol{A}=\left\{\left\langle\bar{\sigma}_{i} \nabla \omega_{j}, \nabla \omega_{k}\right\rangle\right\}_{j, k=1}^{N}$ is the stiffness matrix, $\boldsymbol{v}_{0}, \boldsymbol{w}_{0}$ are spatial discretizations of the initial values, and the components of the vector $\boldsymbol{I}_{i o n}(\boldsymbol{v}, \boldsymbol{w})$ are given by

$$
\boldsymbol{I}_{i o n}(\boldsymbol{v}, \boldsymbol{w})_{k}=\left\langle I_{i o n}\left(\sum_{j=1}^{N} v_{j}(t) \omega_{j}(x), \sum_{j=1}^{N} w_{j}(t) \omega_{j}(x)\right), \omega_{k}\right\rangle .
$$

Analogously, let us consider the adjoint system in its weak formulation:

$$
\begin{aligned}
& \left\langle\frac{\partial p}{\partial t}-\frac{\partial I_{i o n}}{\partial v}(\hat{v}, \hat{w}) p-\frac{\partial G}{\partial v}(\hat{v}, \hat{w}) q-\mathbb{1}_{\Omega_{o b s}} \cdot \hat{v}, \psi\right\rangle=\left\langle\bar{\sigma}_{i} \nabla p, \nabla \psi\right\rangle \forall \psi \in W^{1,2}(\Omega)(\forall) t \in[0, T] \\
& \left\langle\frac{\partial q}{\partial t}, \psi\right\rangle=\left\langle\frac{\partial G}{\partial w}(\hat{v}, \hat{w}) q+\frac{\partial I_{i o n}}{\partial w}(\hat{v}, \hat{w}) p, \psi\right\rangle \quad \forall \psi \in L^{2}(\Omega)(\forall) t \in[0, T] \\
& p(x, T)=0(\forall) x \in \Omega ; \quad q(x, T)=0(\forall) x \in \Omega
\end{aligned}
$$

Inserting the approximate solutions $p(x, t) \approx \sum_{j=1}^{N} p_{j}(t) \omega_{j}(x)$ and $q(x, t) \approx \sum_{j=1}^{N} q_{j}(t) \omega_{j}(x)$ into $(4.7)-$ (4.9), we get the semi-discrete form of the adjoint equations, namely

$$
\begin{aligned}
& \boldsymbol{M} \frac{d \boldsymbol{p}}{d t}=\boldsymbol{A} \boldsymbol{p}+\frac{\partial I_{i o n}}{\partial v}(\boldsymbol{v}, \boldsymbol{w}) \boldsymbol{M} \boldsymbol{p}-\frac{\eta_{2}}{v_{p k}} \boldsymbol{M} \boldsymbol{q}-\boldsymbol{M}_{o b s} \boldsymbol{v} \\
& \frac{d \boldsymbol{q}}{d t}=\eta_{2} \eta_{3} \boldsymbol{q}+\frac{\partial I_{i o n}}{\partial w}(\boldsymbol{v}, \boldsymbol{w}) \boldsymbol{p} ; \quad \boldsymbol{p}(T)=\mathfrak{o} ; \quad \boldsymbol{q}(T)=\mathfrak{o}
\end{aligned}
$$

Here $\boldsymbol{p}=\boldsymbol{p}(t)$ and $\boldsymbol{q}=\boldsymbol{q}(t)$ are the vectors of the Ritz coefficients, $\boldsymbol{M}_{\text {obs }}=\left\{\mathbb{1}_{\Omega_{o b s}} \cdot\left\langle\omega_{j}, \omega_{k}\right\rangle\right\}_{j, k=1}^{N}$ is the restriction of the mass matrix to the observation domain, extended by zero outside, and the numbers $\partial I_{i o n}(\boldsymbol{v}, \boldsymbol{w}) / \partial v$ and $\partial I_{i o n}(\boldsymbol{v}, \boldsymbol{w}) / \partial w$ are given by

$$
\frac{\partial I_{i o n}}{\partial v}(\boldsymbol{v}, \boldsymbol{w})=\frac{\partial I_{i o n}}{\partial v}\left(\sum_{j=1}^{N} v_{j}(t) \omega_{j}(x), \sum_{j=1}^{N} w_{j}(t) \omega_{j}(x)\right) ; \quad \frac{\partial I_{i o n}}{\partial w}(\boldsymbol{v}, \boldsymbol{w})=\eta_{1}\left(\sum_{j=1}^{N} v_{j} \omega_{j}\right) .
$$

Consequently, the semi-discretization of the primal and adjoint equations results in a system of four coupled ODEs, depending on the spatial variables as a parameter, together with initial and terminal conditions.

\section{b) Solution of the coupled ODE system.}

Assume that $\boldsymbol{I}_{e}^{(k)}=\left(I_{e, j}^{(k)}(t)\right)_{j=1}^{N}$ corresponds to a given approximation $I_{e}{ }^{(k)}(x, t) \sum_{j=1}^{N} I_{e, j}^{(k)}(t) \omega_{j}(x)$ for the control variable. Then, by modification of a well-established approach from the numerics of the bidomain equations, ${ }^{22)}$ we decouple the equations within the system (4.4), (4.5), (4.10), (4.11) as follows. To solve first $(4.4)-(4.5)$ at a given spatial point $x$ in forward direction, let us assume that the values $\boldsymbol{v}^{(k),(n)}=\boldsymbol{v}^{(k)}\left(t^{(n)}\right)$ and $\boldsymbol{w}^{(k),(n)}=\boldsymbol{w}^{(k)}\left(t^{(n)}\right)$ have been already calculated. Then we get first $\boldsymbol{w}^{(k),(n+1)}=\boldsymbol{w}^{(k)}\left(t^{(n+1)}\right)$ by an explicit Euler step as

$$
\boldsymbol{w}^{(k),(n+1)}=\boldsymbol{w}^{(k),(n)}+\Delta t\left(\frac{\eta_{2}}{v_{p k}} \boldsymbol{v}^{(k),(n)}-\eta_{2} \eta_{3} \boldsymbol{w}^{(k),(n)}\right),
$$

22) We refer e. g. to [Plank/Liebmann/Weber dos Santos/Vigmond/HaAse 07] and [Trangenstein/Kim 04 ]. 
and subsequently $\boldsymbol{v}^{(k),(n+1)}=\boldsymbol{v}^{(k)}\left(t^{(n+1)}\right)$ is obtained from the ODE

$$
\boldsymbol{M} \frac{d \boldsymbol{v}^{(k)}}{d t}=-\boldsymbol{A} \boldsymbol{v}^{(k)}-\boldsymbol{I}_{i o n}\left(\boldsymbol{v}^{(k)}, \boldsymbol{w}^{(k),(n+1)}\right)+\boldsymbol{I}_{e}^{(k)},
$$

by performing one step of a linearly implicit Runge-Kutta method, namely a Rosenbrock method. ${ }^{23)}$ In our computations, the method ROS2 has been employed. ${ }^{24)}$ The realization of this method results in a system of linear equations, to which an iterative Krylov solver is applied. In the present study, the Bi-CGSTAB method ${ }^{25)}$ with Jacobi preconditioning is used.

Now we turn to the backward solution of $(4.10)-(4.11)$ at $x$. Again, assuming that $\boldsymbol{p}^{(k),(n+1)}=\boldsymbol{p}^{(k)}\left(t^{(n+1)}\right)$ and $\boldsymbol{q}^{(k),(n+1)}=\boldsymbol{q}^{(k)}\left(t^{(n+1)}\right)$ are already known, we calculate first $\boldsymbol{q}^{(k),(n)}=\boldsymbol{q}^{(k)}\left(t^{(n)}\right)$ by

$$
\boldsymbol{q}^{(k),(n)}=\boldsymbol{q}^{(k),(n+1)}-\Delta t\left(\eta_{2} \eta_{3} \boldsymbol{q}^{(k),(n+1)}+\frac{\partial I_{i o n}}{\partial w}\left(\boldsymbol{v}^{(k),(n+1)}, \boldsymbol{w}^{(k),(n+1)}\right) \boldsymbol{p}^{(k),(n+1)}\right),
$$

and then $\boldsymbol{p}^{(k),(n)}=\boldsymbol{p}^{(k)}\left(t^{(n)}\right)$ is obtained from the ODE

$$
\boldsymbol{M} \frac{d \boldsymbol{p}^{(k)}}{d t}=\boldsymbol{A} \boldsymbol{p}^{(k)}+\frac{\partial I_{i o n}}{\partial v}\left(\boldsymbol{v}^{(k),(n+1)}, \boldsymbol{w}^{(k),(n+1)}\right) \boldsymbol{M} \boldsymbol{p}^{(k)}-\frac{\eta_{2}}{v_{p k}} \boldsymbol{M} \boldsymbol{q}^{(k),(n+1)}-\boldsymbol{M}_{o b s} \boldsymbol{v}^{(k),(n+1)}
$$

by application of one step of the Rosenbrock method described above. We end up with a quadruple $\left(\boldsymbol{v}^{(k)}, \boldsymbol{w}^{(k)}\right.$, $\left.\boldsymbol{p}^{(k)}, \boldsymbol{q}^{(k)}\right)$, which corresponds to the current approximation $\boldsymbol{I}_{e}^{(k)}$ for the optimal control.

\section{c) Iterative solution of the optimality system.}

The solution of the optimality system will be obtained by application of an inexact Newton method to the reduced cost functional (3.22). ${ }^{26)}$ The criterion for the termination of this algorithm after the $k$ th iteration is

$$
\left\|\delta^{+} \widetilde{F}\left(I_{e}{ }^{(k)}\right)\right\|_{L^{2}\left(\Omega_{c o n} \times[0, T]\right)} \leqslant 10^{-3} \cdot\left(1+\left|\widetilde{F}\left(I_{e}{ }^{(k)}\right)\right|\right) \quad \text { or } \quad\left|\widetilde{F}\left(I_{e}{ }^{(k)}\right)-\widetilde{F}\left(I_{e}{ }^{(k-1)}\right)\right| \leqslant 10^{-5}
$$

which, in view of (3.23), corresponds to the fulfillment of the optimality condition (3.21) provided that the calculated control $I_{e}{ }^{(k)}(x, t)=\sum_{j=1}^{N} I_{e, j}^{(k)}(t) \omega_{j}(x)$ is feasible. The algorithm may be summarized as follows: Step 0. Initialize $\boldsymbol{I}_{e}^{(1)}$ and $k \leftarrow 1$.

Step 1. Using $\boldsymbol{I}_{e}^{(k)}$, calculate $\boldsymbol{w}^{(k)}$ and $\boldsymbol{v}^{(k)}$ by solving (4.13) and (4.14) forward in time.

Step 2. Using $\boldsymbol{w}^{(k)}$ and $\boldsymbol{v}^{(k)}$, calculate $\boldsymbol{q}^{(k)}$ and $\boldsymbol{p}^{(k)}$ by solving (4.15) and (4.16) backward in time.

Step 3. Update the approximation of the control by performing the inexact Newton step mentioned above, set $\boldsymbol{I}_{e}^{(k)} \leftarrow \boldsymbol{I}_{e}^{(k+1)}$.

Step 4. If $\boldsymbol{I}_{e}{ }^{(k)}$ satisfies (4.17) then terminate else set $k \leftarrow k+1$ and go to Step 1.

In the experiments below, the feasibility of the optimal control has been confirmed a posteriori.

\section{d) The receding horizon strategy.}

In order to allow for longer control horizons without excessively prolonging computing times we use a receding horizon strategy, which can also be thought of as a time-domain decomposition technique. For this purpose the time interval $[0, T]$ is subdivided in subdomains according to $\left[T_{i}, T_{i+1}\right]$ with $i=0, \ldots, n$, where $T_{i}=i T / n$. The receding horizon strategy then consists in successively solving optimal control problems (3.1) - (3.6) with $[0, T]$ replaced by $\left[T_{i}, T_{i+1}\right]$, where, for $i=0, \ldots, n$, the initial conditions (3.4) $-(3.5)$ are chosen such that the optimal states at $t=T_{i}$ from the domain $\left[T_{i-1}, T_{i}\right]$ are taken as initial condition for the time interval

23) Cf. [HAIRER/WANner 02], pp. $102 \mathrm{ff}$.

24) [LANG 01] , pp. $47-57$.

25) Introduced first in [VAN DER VORST 92], this became a standard method.

26) A detailed description of the method may be found in [NAGAIAH/KUNISCH 11], pp. 56 f. and 65. 
$\left[T_{i}, T_{i+1}\right]$. While this procedure violates the Bellman principle and will not give the optimal solution for the global problem on $[0, T]$, it can be shown that under appropriate conditions it is effective in achieving the design objective. ${ }^{27)}$ In our case this objective will consist in the termination of a re-entrant wave.

\section{e) Parallel implementation.}

In the numerical treatment of the monodomain as well as of the full bidomain equations, parallelization has been proven to be an efficient approach in order to achieve acceptable computational times for realistic simulations. ${ }^{28)}$ It turns out that the optimization method described above is well-suited for parallelization as well. The localized structure of the method allows for a spatial domain decomposition within the solution of the primal and adjoint equations as well as within the solution of their linearizations required for the calculation of the inexact Newton update.

For the implementation, we used the package $D U N E,{ }^{29)}$ a $C++$ based programming environment for the solution of different classes of PDEs. For the parallel grid generation, the internal parallel Yasp grid has been used, which supports various levels of overlapping grids. In our calculations, we used a zero level, i. e., nonoverlapping grids. The subdomains were assigned to the processors in a one-to-one mapping. Our discretization routines are based on the DUNE-PDELab discretization module. The coupling of the subdomains through the values of the unknown solutions at the interfaces has been further relaxed by introduction of additional communication steps during the solution of the algebraic systems. In the parallel computations, the above mentioned Bi-CGSTAB method with Jacobi preconditioning has been used. The complete parallel algorithm is given in the appendix.

\section{Numerical results.}

\section{a) Dampening of an excitation wave: parallel efficiency.}

We choose $\Omega=[0,1] \times[0,1] \subset \mathbb{R}^{2}$ and $T=4$. The control domain $\Omega_{c o n}=\Omega_{c o n, 1} \cup \Omega_{c o n, 2}$ consists of the disjoint areas $\Omega_{c o n, 1}=[0.320,0.400] \times[0.450,0.550]$ and $\Omega_{c o n, 2}=[0.600,0.680] \times[0.450,0.550]$. The observation domain is obtained as the complement of a neighborhood of $\Omega_{\text {con }}$. More precisely, defining $\widetilde{\Omega}_{c o n, 1}=[0.280,0.420] \times[0.430,0.570]$ and $\widetilde{\Omega}_{c o n, 2}=[0.580,0.720] \times[0.450,0.550]$, we take $\Omega_{o b s}=$ $\Omega \backslash\left(\widetilde{\Omega}_{c o n, 1} \cup \widetilde{\Omega}_{c o n, 2}\right)$. The positions of the subdomains are depicted in Figure 5.1. Moreover, the regularization parameter is chosen as $\alpha=0.0006$, and the uniform bound for the controls as $R=2000$. For the intracellular conductivity tensor, the constant matrix ${ }^{30)}$

$$
\bar{\sigma}_{i}(x) \equiv\left(\begin{array}{cc}
3 \cdot 10^{-3} & 0 \\
0 & 3.1525 \cdot 10^{-4}
\end{array}\right)
$$

has been chosen. Within the Rogers-McCulloch model, the parameters are specified as follows: $\eta_{0}=1.5$, $\eta_{1}=4.4, \eta_{2}=0.012, \eta_{3}=1.0, v_{t h}=13$ and $v_{p k}=100$. The quantities are given in the following units: $[T]=$ $[\Delta t]=\mathrm{ms},\left[\left(\bar{\sigma}_{i}\right)_{k l}\right]=\Omega^{-1} \mathrm{~cm}^{-1},\left[\eta_{0}\right]=\left[\eta_{1}\right]=\mathrm{S} \mathrm{cm}^{-2},\left[\eta_{2}\right]=\left[\eta_{3}\right]=1,[v]=\left[v_{0}\right]=\left[v_{t h}\right]=\left[v_{p k}\right]=\mathrm{mV}$, $[w]=1$ and $\left[I_{e}\right]=[R]=\mu \mathrm{A}$. The excitation wave is modeled by the choice of the initial values

$$
v_{0}(x)=101.00 \cdot \mathbb{1}_{\Omega_{e x c}}(x) ; \quad w_{0}(x) \equiv 0,
$$

where $\Omega_{e x c}=[0.498,0.502] \times[0.498,0.504] \subset \Omega_{o b s}$.

27) [Chen/Allgöwer 98] and [Ito/Kunisch 02]

28) See e. g. [Colli Franzone/Pavarino 04], [Pavarino/Scacchi 08], [Plank/Liebmann/Weber dos Santos/ Vigmond/Hahe 07] and [Vigmond/Boyle/Leon/Plank 09].

29) Described in [Bastian/Blatt/Dedner/Engwer/Klöfkorn/Kornhuber/Ohlberger/Sander 08] .

30) These values as well as the parameters for the Rogers-McCulloch model have been taken from [ColLi Franzone/Deuflhard/Erdmann/Lang/Pavarino 06], p. 952, Table 4.1. Note that the same parameters have been used within the experiments in [NAGAIAH/Kunisch 11]. 
For the discretization, we used a uniform quadrilateral spatial grid with $129 \times 129$ nodes and 100 time steps with uniform length $\Delta t=0.04$. The parallel computations have been carried out with the cluster GHOST located at the TU Graz. ${ }^{31)}$ It is a 64-bit Linux cluster with two nodes, each of them consisting of 8 quad-core AMD Opteron processors $8356(2.3 \mathrm{GHz})$ with $256 \mathrm{~GB}$ RAM, a dedicated gigabit ethernet link for the shared file system and two bundled gigabit ethernet links for the communication between the parallel jobs. In the calculations, only a single node has been used. ${ }^{32}$ )

The results of our experiments will be summarized in Table 5.1. The average of the CPU time elapsed for a single primal solve and the CPU time for the first calculation of the Newton update, respectively, will be shown in columns 2 and 4 while the total CPU time consumed for the solution of $(\mathrm{P})$ will be shown in column 6. We give the CPU times in percents related to the single-processor experiment. Besides, we show the parallel efficiency according to $e=T(1) /(N T(N))$ where $N$ is the number of processors.

\begin{tabular}{|c|c|c|c|c|c|c|}
\hline & \multicolumn{2}{|c|}{ single primal solve } & \multicolumn{2}{|c|}{ first Newton step } & \multicolumn{2}{|c|}{ complete solution } \\
\hline$N$ & CPU time (aver.) & efficiency & CPU time & efficiency & CPU time (total) & efficiency \\
\hline 1 & 100.0 & 1.0000 & 100.0 & 1.0000 & 100.0 & 1.0000 \\
\hline 2 & 52.0 & 0.9619 & 52.3 & 0.9563 & 54.1 & 0.9239 \\
\hline 4 & 26.2 & 0.9539 & 26.6 & 0.9395 & 29.2 & 0.8557 \\
\hline 8 & 13.2 & 0.9434 & 13.6 & 0.9186 & 15.4 & 0.8116 \\
\hline 16 & 6.7 & 0.9372 & 6.9 & 0.9084 & 7.9 & 0.7933 \\
\hline 32 & 3.4 & 0.9102 & 3.5 & 0.8813 & 4.2 & 0.7346 \\
\hline
\end{tabular}

\section{b) Termination of a re-entrant wave.}

In our second experiment, we work on the domain $\Omega=[0,2] \times[0,2] \subset \mathbb{R}^{2}$. According to the receding horizon strategy, the initial endtime $T_{1}=4$ has been increased in steps of $\Delta T=4$ until $T_{45}=T=180$. The control domain $\Omega_{\text {con }}=\Omega_{\text {con,1 }} \cup \Omega_{\text {con,2 }}$ consists again of two disjoint parts, namely the strips $\Omega_{\text {con,1 }}=$ $[0.400,0.500] \times[0.000,0.650]$ and $\Omega_{\text {con, } 2}=[1.300,1.400] \times[0.000,1.000]$. Defining the neighborhoods $\widetilde{\Omega}_{c o n, 1}=[0.350,0.550] \times[0.000,0.700]$ and $\widetilde{\Omega}_{c o n, 2}=[1.250,1.450] \times[0.000,1.050]$, the observation domain will be described as $\Omega_{o b s}=\Omega \backslash\left(\widetilde{\Omega}_{c o n, 1} \cup \widetilde{\Omega}_{c o n, 2}\right)$. The positions of the subdomains will be shown in Fig. 5.1. (b) ( $\Omega_{\text {obs }}$ in blue, $\Omega_{\text {con }}$ in red, $\Omega_{\text {exc }}$ is not depicted). We take the regularization parameter $\alpha=0.001$ and the control bound $R=2000$. The values of $\bar{\sigma}_{i}$ as well as of the parameters within the Rogers-McCulloch model are the same as in Subsection 5.a). In order to generate an initial state, which develops into a re-entrant wave without application of a control, we calculated a numerical solution $(\tilde{v}, \tilde{w})$ of the monodomain system with initial values

$$
\tilde{v}_{0}(x)=101.00 \cdot \mathbb{1}_{\Omega e x c}(x) ; \quad \tilde{w}_{0}(x) \equiv \mathfrak{o}
$$

and the excitation domain $\Omega_{e x c}=[0,2] \times[0,0.002]$ on the time interval $[0,450]$ according to the strategy described in Subsection 4.b) but using variable time steps. ${ }^{33)}$ Our experiment has now been initialized with

$$
v_{0}(x)=\tilde{v}(x, 450) ; \quad w_{0}(x)=\tilde{w}(x, 450)
$$

31) An online documentation is available at http://ghost.tugraz.at/wiki/index.php/Main_Page (last accessed: 17.02.2011).

32) Due to the significant loss of performance caused by the low speed of the gigabit communication links between two nodes.

33) Cf. [LANG 01], p. 32 f. 
(see Fig. 5.2.). In the discretization, again a uniform quadrilateral spatial grid with $129 \times 129$ nodes and uniform time steps with length $\Delta t=0.04$ have been used. The calculations have been performed on the cluster GHOST described above. For comparison, the evolution of the uncontrolled solution $(\tilde{v}(x, 450+t), \tilde{w}(x, 450+t))$ has been simulated as well.

In Fig. 5.3., we show in the left column some snapshots of the development of the uncontrolled transmembrane potential $\tilde{v}$. The re-entrant wavefront starts at the center of $\Omega$ and leaves the domain at the boundaries. In the middle column, we oppose to $\tilde{v}$ the controlled state $v$. Even after a simulation time of $113 \mathrm{~ms}$, the excitation wave has only been partly removed. Its complete removal has been achieved at a simulation time of $180 \mathrm{~ms}$. The optimal control in the right column shows sharp peaks but remains inactive during the experiment. The complete simulation, comprising 45 time frames, took approximately $1.25 \mathrm{~h}$ of CPU time using 32 processors.

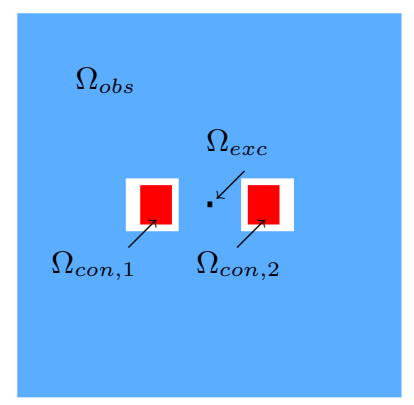

(a) First experiment.

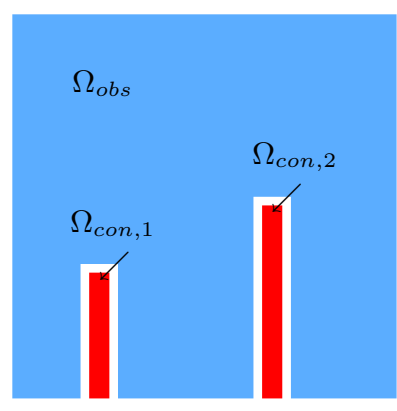

(b) Second experiment.

Fig. 5.1. Locations of the subdomains in the first and second experiment.

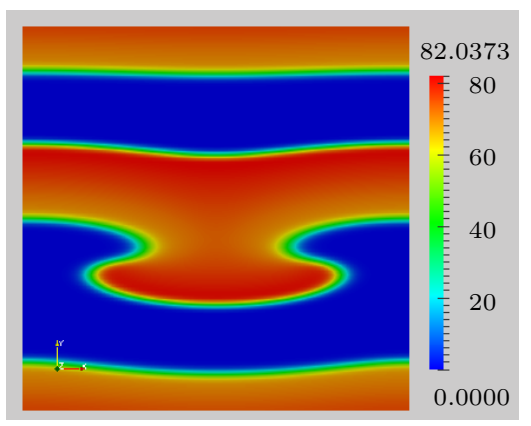

(a) $v_{0}(x)=\tilde{v}(x, 450)$.

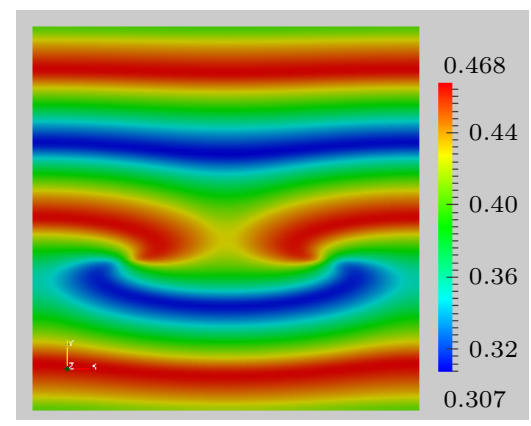

(b) $w_{0}(x)=\tilde{w}(x, 450)$.

Fig. 5.2. Initial state for the second experiment, corresponding to a re-entrant wave.

\section{c) Discussion and conclusions.}

The numerical experiments showed that the optimal control approach is successful with respect to achieving the design objective of dampening excitation waves and reentry phenomena. Special attention was payed to the fact that the control domain is chosen to be small relative to the physiological domain. For the overall optimization strategy, the computation time has been reduced through parallelization to less than $5 \%$ compared to the sequential code, while the parallel efficiency ranges about $e=0.75$. This is an essential step towards the treating of the full bidomain model and carrying out optimal control computations in three-dimensional spatial domains. Even with parallelization, the use of the receding horizon strategy is unevitable for carrying out numerical optimal control of this kind of systems over time horizons of physiological relevance. We have tested the stability of the employed receding horizon strategy by applying the computed optimal controls to the monodomain system, which was perturbed by Gaussian noise added to the right-hand side of the parabolic equations. Still, the design objectives could be achieved. 
Clearly many problems remain open for future work on this challenging problem. These include the investigation of the effects of anisotropy on the overall control mechanism, more realistic gating models and geometries, and relating mathematically imposed constraints, as for instance control constraints, to their physiological scale and relevance.

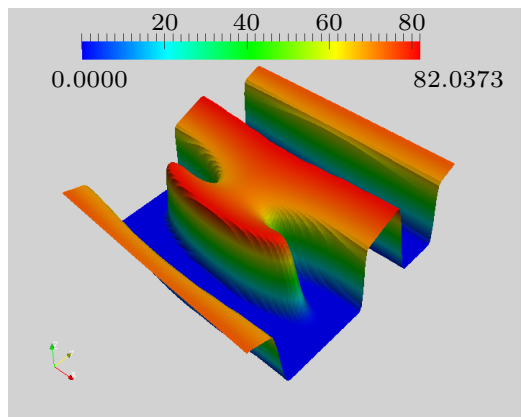

(a) $t=1.88 \mathrm{~ms}$

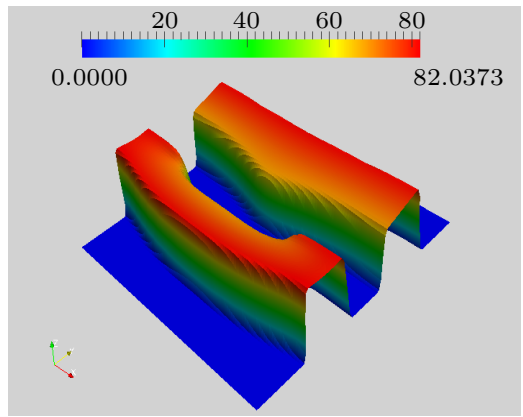

(d) $t=22.68 \mathrm{~ms}$

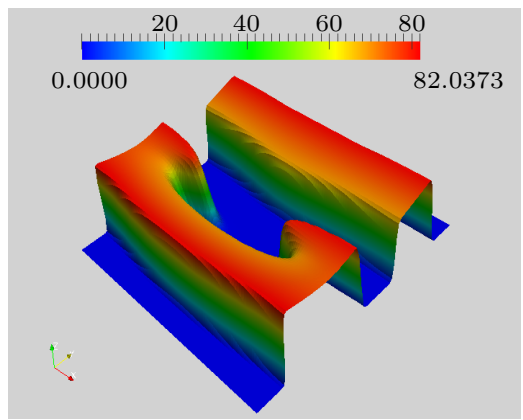

(g) $t=113.36 \mathrm{~ms}$

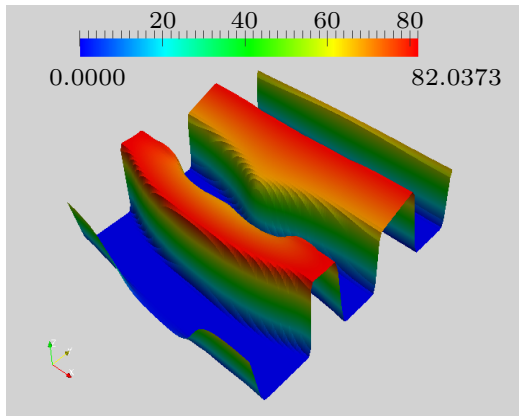

(j) $t=179.48 \mathrm{~ms}$

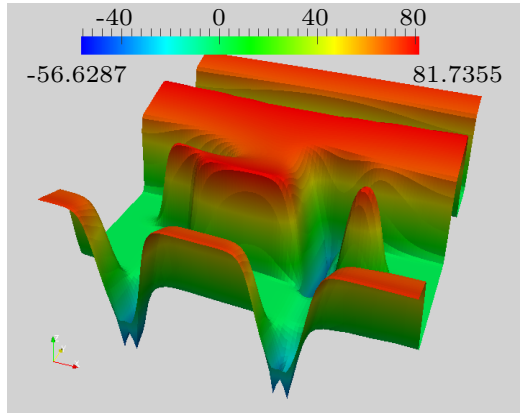

(b) $t=1.88 \mathrm{~ms}$

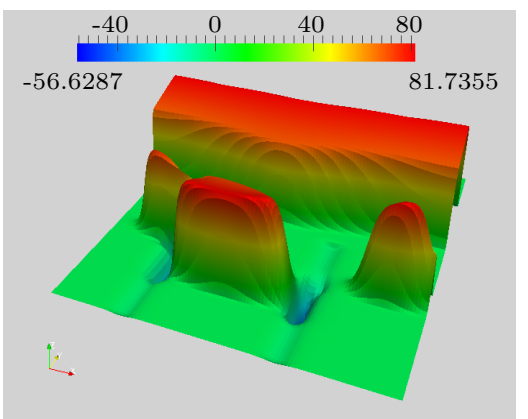

(e) $t=22.68 \mathrm{~ms}$

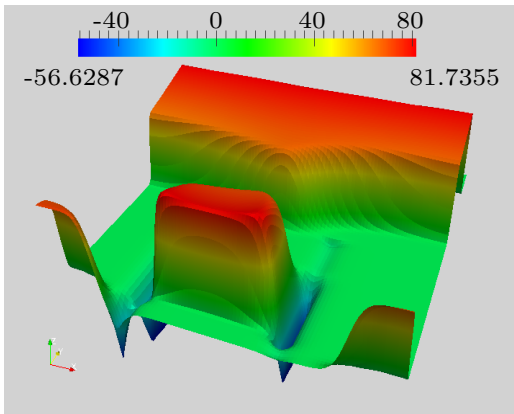

(h) $t=113.36 \mathrm{~ms}$

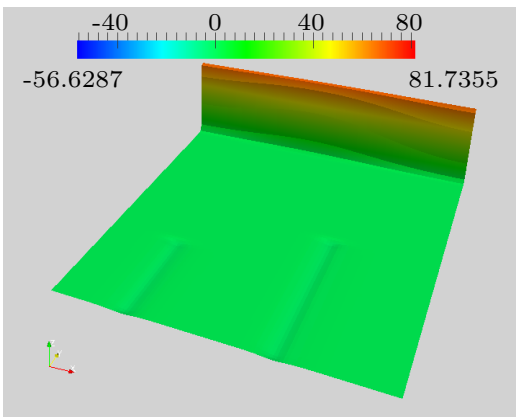

(k) $t=179.48 \mathrm{~ms}$

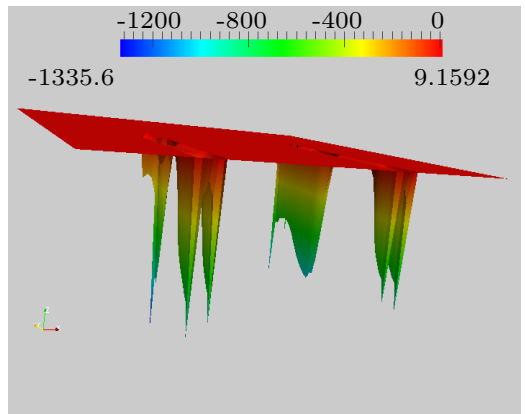

(c) $t=1.88 \mathrm{~ms}$

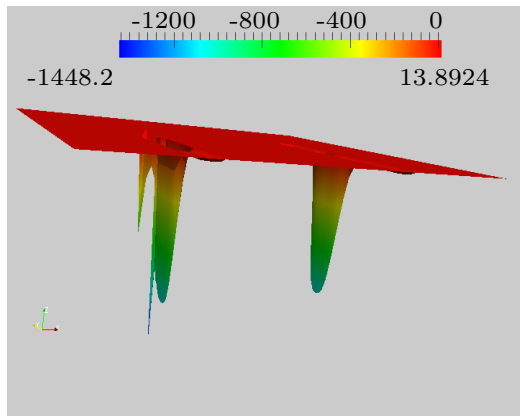

(f) $t=22.68 \mathrm{~ms}$

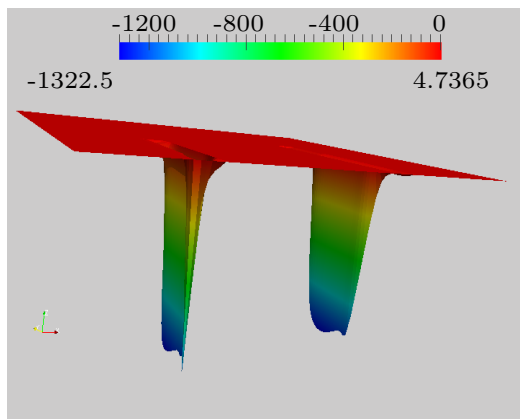

(i) $t=113.36 \mathrm{~ms}$

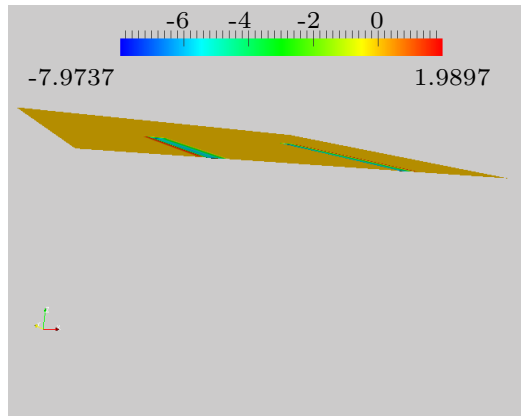

(l) $t=179.48 \mathrm{~ms}$

Fig. 5.3. Left: transmembrane potential $\tilde{v}(450+t)$, uncontrolled. Middle: transmembrane potential $\hat{v}(t)$, controlled. Right: optimal control $\hat{I}_{e}(t)$. 


\section{Acknowledgement.}

The present work has been supported within the Special Research Unit "Mathematical Optimization and Applications in Biomedical Sciences" (Graz) by the Austrian Science Fund.

\section{Appendix.}

In the statement of the complete optimization algorithm below, ${ }^{34)}$ the parallel communication steps are explicitly mentioned.

\section{Algorithm 1. Parallel line search Newton-CG optimization algorithm.}

1: primal variables $\boldsymbol{v}, \boldsymbol{w}$; dual variables $\boldsymbol{p}, \boldsymbol{q}$; control variable $\boldsymbol{I}_{e}$

2: initialize $\boldsymbol{I}_{e}^{(1)}$ and $k \leftarrow 1$

3: repeat

4: $\quad$ set $\boldsymbol{w}(0)=\boldsymbol{w}_{0}, \boldsymbol{v}(0)=\boldsymbol{v}_{0}$; calculate $\boldsymbol{w}^{(k)}$ and $\boldsymbol{v}^{(k)}$ by solving (4.13) - (4.14) forward in time, using $\boldsymbol{I}_{e}{ }^{(k)}$ (communication needed at solution of algebraic equations)

5: $\quad$ set $\boldsymbol{q}(T)=0, \boldsymbol{p}(T)=0$; calculate $\boldsymbol{q}^{(k)}$ and $\boldsymbol{p}^{(k)}$ by solving (4.15) - (4.16) backward in time, using $\boldsymbol{w}^{(k)}$ and $\boldsymbol{v}^{(k)}$ (communication needed at solution of algebraic equations)

6: update the gradient $\nabla \widetilde{F}^{(k)}=-\boldsymbol{p}^{(k)}+\alpha \boldsymbol{I}_{e}{ }^{(k)}$, using the adjoint solution (no communication) and solve the system $\nabla^{2} \widetilde{F}^{(k)} \Delta \boldsymbol{I}_{e}=-\nabla \widetilde{F}^{(k)}$ by a linear CG method as follows:

7: repeat

the CG iteration step with the following matrix-vector product procedure: ${ }^{35)}$

8: $\quad$ solve the linearized primal equations (communication needed at solution of algebraic equations)

9: $\quad$ evaluate the second derivative $\nabla^{2} \widetilde{F}^{(k)}$

10: $\quad$ solve the linearized adjoint equations (communication needed at solution of algebraic equations)

11: compute the action of the Hessian $\nabla^{2} \widetilde{F}^{(k)}$ on $\Delta \boldsymbol{I}_{e}$ (two more communications per CG step are needed in order to find the scalar product)

12: $\quad$ until residuum $<\operatorname{Min}\left(10^{-3},\left\|\nabla \widetilde{F}^{(k)}\right\|_{L^{2}\left(\Omega_{c o n} \times[0, T]\right)}\right) \cdot\left\|\nabla \widetilde{F}^{(k)}\right\|_{L^{2}\left(\Omega_{c o n} \times[0, T]\right)}$

13: set step length $\beta^{(k)}=1$ and compute optimal $\beta^{(k)}$ using backtracking method by checking the strong Wolfe conditions ${ }^{36)}$ (communication needed at solution of algebraic equations as well as for evaluation of the cost functional)

14: update $\boldsymbol{I}_{e}^{(k+1)} \leftarrow \boldsymbol{I}_{e}^{(k)}+\beta^{(k)} \Delta \boldsymbol{I}_{e}$, using optimal $\beta^{(k)}$

15: $\quad k \leftarrow k+1$

16: until $\left\|\nabla \widetilde{F}^{(k)}\right\|_{L^{2}\left(\Omega_{c o n} \times[0, T]\right)} \leqslant 10^{-3} \cdot\left(1+\left|\widetilde{F}^{(k)}\right|\right) \quad$ or $\quad\left|\widetilde{F}^{(k)}-\widetilde{F}^{(k-1)}\right| \leqslant 10^{-5}$ (communication needed for evaluation of the cost functional)

34) We refer to [Nagaiah/Kunisch 11], p. 65, Algorithm 1.

35) The following steps 8-11 correspond to [NAGAiah/Kunisch 11], p. 57, Steps (a) - (d).

36) [Nocedal/Wright 06], pp. $33 \mathrm{ff}$. 


\section{References.}

1. [Anderson/Trayanova/Skouibine 00] Anderson, C.; Trayanova, N.; Skouibine, K.: Termination of spiral waves with biphasic shocks: role of virtual electrode polarization. J. Cardiovasc. Electrophysiol. 11 (2000), 1386 - 1396

2. [Bastian/Blatt/Dedner/Engwer/Klöfkorn/Kornhuber/Ohlberger/Sander 08] Bastian, P.; Blatt, M.; Dedner, A.; Engwer, C.; Klöfkorn, R.; Kornhuber, R.; Ohlberger, M.; Sander, O.: A generic grid interface for parallel and adaptive scientific computing. II. Implementation and tests in DUNE. Computing 82 (2008), 121 - 138

3. [Biegler/Ghattas/Heinkenschloss/Keyes/van Bloemen WaAnders 07] Biegler, L. T.; Ghattas, O.; Heinkenschloß, M.; Keyes, D.; van Bloemen Waanders, B. (Eds.): it Real-Time PDE-Constrained Optimization. SIAM; Philadelphia 2007

4. [Biros/Ghattas 05] Biros, G.; Ghattas, O.: Parallel Lagrange-Newton-Krylov-Schur methods for PDE-constrained optimization. I. The Krylov-Schur solver. SIAM J. Sci. Comput. 27 (2005), $687-713$

5. [Bourgault/Coudière/Pierre 09] Bourgault, Y.; Coudière, Y.; Pierre, C.: Existence and uniqueness of the solution for the bidomain model used in cardiac electrophysiology. Nonlinear Analysis: Real World Appl. 10 (2009), $458-482$

6. [Brandẽo/Fernández-Cara/Magalhães/Rojas-Medar 08] Brandaõ, A. J. V.; Fernández-Cara, E.; Magalhães, P. M. D.; Rojas-Medar, M. A.: Theoretical analysis and control results for the FitzHugh-Nagumo equation. Electronic J. of Differential Equations, Vol. 2008, No. 164, 1 - 20 (electronically published)

7. [Chen/Allgöwer 98] Chen, H.; Allgöwer, F.: A quasi-infinite horizon nonlinear model predictive control scheme with guaranteed stability. Automatica J. IFAC 34 (1998), 1205 - 1217

8. [ Colli Franzone/Deuflhard/Erdmann/Lang/Pavarino 06] Colli Franzone, P.; Deuflhard, P.; Erdmann, B.; Lang, J.; Pavarino, L. F.: Adaptivity in space and time for reaction-diffusion systems in electrocardiology. SIAM J. Sci. Computing 28 (2006), 942 - 962

9. [Colli Franzone/Pavarino 04] Colli Franzone, P.; Pavarino, L. F.: A parallel solver for reaction-diffusion systems in computational electrocardiology. Math. Models and Methods in Appl. Sci. 14 (2004), 883 - 911

10. [Evans 98] Evans, L. C.: Partial Differential Equations. American Mathematical Society; Providence 1998

11. [Hairer/Wanner 02] Hairer, E.; Wanner, G.: Solving Ordinary Differential Equations. Vol. II. Springer; Berlin etc. 2002 , 2nd ed.

12. [Hintermüller/Hoppe 08] Hintermüller, M.; Hoppe, R. H. W.: Goal-oriented adaptivity in control constrained optimal control of partial differential equations. SIAM J. Control Optim. 47 (2008), 1721 - 1743

13. [Hinze/Kunisch 01] Hinze, M.; Kunisch, K.: Second order methods for optimal control of time-dependent fluid flow. SIAM J. Control Optim. 40 (2001), 925 - 946

14. [ Ito/Kunisch 02] Ito, K.; Kunisch, K.: Receding horizon optimal control for infinite dimensional systems. ESAIM Control Optim. Calc. Var. 8 (2002), $741-760$

15. [ Ito/Kunisch 08] Ito, K.; Kunisch, K.: Lagrange Multiplier Approach to Variational Problems and Applications. SIAM; Philadelphia 2008

16. [Keener/Sneyd 09] Keener, J.; Sneyd, J.: Mathematical Physiology. Vol. II: Systems Physiology. Springer; New York etc. 2009, 2nd ed.

17. [Kunisch/Wagner 11] Kunisch, K.; Wagner, M.: Optimal control of the bidomain system (I): The monodomain approximation with the Rogers-McCulloch model. SFB-Report 2011-001, Institute for Mathematics and Scientific Computing, University of Graz 2011

18. [LAng 01] Lang, J.: Adaptive Multilevel Solution of Nonlinear Parabolic PDE Systems. Springer; Berlin etc. 2001 (Lecture Notes in Computational Science and Engineering 16)

19. [Meidner/Vexler 07] Meidner, D.; Vexler, B.: Adaptive space-time finite element methods for parabolic optimization problems. SIAM J. Control Optim. 46 (2007), $116-142$

20. [NagAiah/Kunisch 11] Nagaiah, C.; Kunisch, K.: Higher order optimization and adaptive numerical solution for optimal control of monodomain equations in cardiac electrophysiology. Appl. Num. Math. 61 (2011), 53 - 65

21. [Nagaiah/Kunisch/Plank 09] Nagaiah, C.; Kunisch, K.; Plank, G.: Numerical solution for optimal control of the reaction-diffusion equations in cardiac electrophysiology. To appear in: Comp. Optim. Appl.; DOI: 10.1007/s10589009-9280-3 
22. [Nagaiah/Kunisch/Plank 10] Nagaiah, C.; Kunisch, K.; Plank, G.: Numerical solutions for optimal control of monodomain equations in cardiac electrophysiology. In: Diehl, M.; Glineur, F.; Jarlebring, E.; Michiels, W. (Eds.): Recent Advances in Optimization and its Applications in Engineering. Springer; Berlin etc. 2010, 409 - 418

23. [Nielsen/Rudd/Lines/Tveito 07] Nielsen, B. F.; Ruud, T. S.; Lines, G. T.; Tveito, A.: Optimal monodomain approximations of the bidomain equations. Appl. Math. Comput. 184 (2007), $276-290$

24. [Nocedal/Wright 06] Nocedal, J.; Wright, S. J.: Numerical Optimization. Springer; New York etc. 2006, 2nd ed.

25. [PAvarino/ScAcchi 08] Pavarino, L. F.; Scacchi, S.: Multilevel additive Schwarz preconditioners for the bidomain reaction-diffusion system. SIAM J. Sci. Comput. 31 (2008), 420 - 445

26. [Plank/Liebmann/Weber dos Santos/Vigmond/Hahse 07] Plank, G.; Liebmann, M.; Weber dos Santos, R.; Vigmond, E. J.; Haase, G.: Algebraic multigrid preconditioner for the cardiac bidomain model. IEEE Trans. Biomed. Eng. 54 (2007), 585 - 596

27. [Potse/Dubé/Richer/Vinet/Gulrajani 06] Potse, M.; Dubé, B.; Richer, J.; Vinet, A.; Gulrajani, R. M.: A comparison of monodomain and bidomain reaction-diffusion models for action potential propagation in the human heart. IEEE Trans. Biomed. Eng. 53 (2006), 2425 - 2435

28. [Rogers/McCulloch 94] Rogers, J. M.; McCulloch, A. D.: A collocation-Galerkin finite element model of cardiac action potential propagation. IEEE Trans. Biomed. Eng. 41 (1994), 743 - 757

29. [Sundnes/Lines/Cai/Nielsen/Mardal/Tveito 06] Sundnes, J.; Lines, G. T.; Cai, X.; Nielsen, B. F.; Mardal, K.-A.; Tveito, A.: Computing the Electrical Activity in the Heart. Springer; Berlin etc. 2006

30. [Trangenstein/Kim 04] Trangenstein, J. A.; Kim, C.: Operator splitting and adaptive mesh refinement for the Luo-Rudy I model. J. Comput. Physics 196 (2004), 645 - 679

31. [VAN DER VORST 92] van der Vorst, H. A.: Bi-CGSTAB: a fast and smoothly converging variant of Bi-CG for the solution of nonsymmetric linear systems. SIAM J. Sci. Statist. Comput. 13 (1992), 631 - 644

32. [Veneroni 09] Veneroni, M.: Reaction-diffusion systems for the macroscopic bidomain model of the cardiac electric field. Nonlinear Analysis: Real World Applications 10 (2009), 849 - 868

33. [Vigmond/Boyle/Leon/Plank 09] Vigmond, E. J.; Boyle, P. M.; Leon, L.; Plank, G.: Near-real-time simulations of biolelectric activity in small mammalian hearts using graphical processing units. In: Engineering in Medicine and Biology Society, 2009. EMBC 2009. Annual International Conference of the IEEE. IEEE; Minneapolis 2009, $3290-3293$

34. [Vigmond/Weber dos Santos/Prassl/Deo/Plank 08] Vigmond, E. J.; Weber dos Santos, R.; Prassl, A. J.; Deo, M.; Plank, G.: Solvers for the cardiac bidomain equations. Progress in Biophysics \& Molecular Biology 96 (2008), $3-18$

35. [Weber dos Santos/Plank/Bauer/Vigmond 04] Weber dos Santos, R.; Plank, G.; Bauer, S.; Vigmond, E. J.: Parallel multigrid preconditioner for the cardiac bidomain model. IEEE Trans. Biomed. Eng. 51 (2004), 1960 1968

Last modification: 28.02 .2011

Authors' addresses / e-mail. Karl Kunisch: University of Graz, Institute for Mathematics and Scientific Computing, Heinrichstraße 36, A-8010 Graz, Austria. e-mail: karl.kunisch @ uni-graz.at

Chamakuri Nagaiah: University of Graz, Institute for Mathematics and Scientific Computing, Heinrichstraße 36, A-8010 Graz, Austria. e-mail: nagaiah.chamakuri @ uni-graz.at

Marcus Wagner: University of Graz, Institute for Mathematics and Scientific Computing, Heinrichstraße 36, A-8010 Graz, Austria. Homepage / e-mail: www.thecitytocome.de / marcus . wagner @ uni-graz .at 